\title{
Using Social Media to Explore Mental Health-Related Behaviors and Discussions among Young Adults
}

\author{
Moloud Shahbazi MS \\ University of California, Riverside \\ MoloudShahbazi@gmail.com \\ Moloud Shahbazi MS \\ University of California, Riverside \\ MoloudShahbazi@gmail.com
}

\author{
Renee Garett MSW,LCSW \\ ElevateU \\ ReneeGarettLCSW@gmail.com
}

Sean Young $\mathrm{PhD}$, MS

University of CA, Los Angeles

SDYoung@mednet.ucla.edu

\begin{abstract}
There have been recurring reports of online harassment and abuse among adolescents and young adults through Anonymous Social Networking websites (ASNs). We explored discussions related to social and mental health behaviors among college students, including cyberbullying on the popular ASN, Yik Yak. From April 6, 2016, to May 7, 2016, we collected anonymous conversations posted on Yik Yak at 19 universities in four different states. We found that prosocial messages were approximately five times as prevalent as bullying messages. Frequency of cyberbullying messages was positively associated with messages seeking emotional help. We found significant geographic variation in the frequency of messages offering supportive versus bullying messages. Across campuses bullying and political discussion were positively associated. Results suggest that ASN sites can be mined for real-time data about students' mental health-related attitudes and behaviors. We discuss the implications for using this information in education and healthcare services.
\end{abstract}

\section{Introduction}

The transition from high school to college marks an important period of psychosocial development, with significant implications for a healthy and productive adulthood. The academic and social demands of college life are often rigorous and can pose a risk to undergraduate students' health and well-being. A major problem among college freshman, for example, is poor sleep [1], which has been linked to a number of adverse consequences, including higher rates of depressive symptoms and stress [2,3], weight gain [4], and poor academic performance [5]. In recent years, social media use among undergraduate students has become another concern. Studies show a link between cyberbullying and major health problems such as substance use, depression, and suicide [6-8].

Given the array of health risks faced by undergraduate students, it is important to be aware of student health and risk-related behaviors to be able to provide adequate services and support, such as from psychological and medical campus services. Traditionally, methods for monitoring student health have focused on case reports and surveys. Although these methods can offer insights into health-related attitudes and behaviors, they can be time- and costintensive to implement. By using social media data, researchers can collect and analyze behavior data in real time. This allows health authorities to address student needs in a flexible and timely manner.

In order to explore the feasibility of using social media platforms to identify and predict health-related events, Young et al., (2014) screened geolocated Twitter messages for keywords that suggested HIV risk behaviors. The authors used negative binomial regression analyses to determine the association between tweets about HIV risk behaviors and countylevel HIV data in the United States. They ran analyses to determine the association between tweets about HIV risk behaviors and county-level HIV data in the United States. The results showed a strong association between tweets about HIV risk behaviors and actual county HIV data [9]. Additionally, De Choudhury and colleagues successfully used tweets to predict the onset of major depressive disorder with $70 \%$ accuracy. They selected tweets based on indicators such as linguistic style, use of terms associated with depression, and social- network characteristics [10].

Yik Yak was an anonymous online bulletin board for users within the same geographic area (e.g., college campuses) that debuted in 2013. At the time of this study, it was a popular social network for college 
students. Critics of the social network argue, aided by anecdotal evidence relayed through media reports, that anonymous posting encourages harassment and bullying [11-14]. In a recent content analysis of Yik Yak conversations, there was no evidence of a pervasive culture of harassment and abuse. However, contradictory to this analysis, researchers did observe derogatory and incendiary comments, arguably racist and sexist messages, and several likely instances of bullying [15].

In this study, we explored the types of posts students were making on Yik Yak related to social and health behaviors, including cyberbullying. Our goal was to provide insights for school administrators, public health researchers, and health care professionals regarding the prevalence of behaviors such as bullying and social support, as well as knowledge of general topics discussed on the network. Knowledge of these activities on Yik Yak and other Anonymous Social Networks ${ }^{1}$ (ASNs) can inform interventions that promote healthy and prosocial behaviors among adolescents and young adults.

\section{Methods}

From April 6, 2016, to May 7, 2016, we collected anonymous conversations posted on the Yik Yak social network at 19 universities located in California, Florida, New York, and Texas (Table 1). Due to this being a pilot study designed to explore conversations among college students, we arbitrarily chose 19 universities in the U.S. We collected conversations from the 19 universities during a limited time period between April 6 and May 7, 2016. We then randomly selected 100 conversation threads from each of the universities ( $\mathrm{N}=16,966$ messages), with a mean of 893 messages per university $(S D=128)$. We analyzed the messages with respect to the type of messaging behavior, content, and popularity of message type and content.

Table 1. Characteristics of universities included in the

\begin{tabular}{|c|c|c|c|c|}
\hline State & University & $\begin{array}{l}\text { Public/ } \\
\text { Private }\end{array}$ & $\begin{array}{l}\text { Enroll } \\
\text { ment }\end{array}$ & $\begin{array}{l}\text { Rank } \\
\text { ing }\end{array}$ \\
\hline \multirow{6}{*}{ CA } & California Polytechnic & & & \\
\hline & State University & Public & 19,226 & 221 \\
\hline & CSU Chico & Public & 16,535 & 467 \\
\hline & CSU Los Angeles & Public & 20,353 & 700 \\
\hline & CSU San Bernardino & Public & 17,167 & 700 \\
\hline & University of & Public & 25,001 & 153 \\
\hline
\end{tabular}

${ }^{1} \mathrm{ASN}=$ Anonymous Social Network

\author{
California Irvine \\ Florida International \\ FL University \\ Florida State \\ University \\ University of Central \\ Florida \\ University of Florida \\ University of South \\ Florida \\ Public $\quad 53,525 \quad 550$ \\ Public 36,575226 \\ Public $59,894 \quad 445$ \\ Public 36,731 56 \\ Public 35,035 396 \\ NY Cornell University Private 14,706 9 \\ CUNY Hunter College Public 20,582 350 \\ CUNY John Jay \\ College of Criminal \\ Justice \\ SUNY Buffalo State \\ Public $\quad 15,845 \quad 700$ \\ SUNY New Paltz Public 7,756 423 \\ Tarleton State \\ TX University Public $11,008 \quad 800$ \\ Texas Tech University Public 29,342 550 \\ University of Houston Public $36,128 \quad 388$ \\ University of Texas \\ Rio Grande Valley \\ Public 27,560 - \\ Note. Source of enrollment and ranking data: Wall \\ Street Journal/Times Higher Education College \\ Rankings 2017. \\ CA, California; FL, Florida; NY, New York; TX, \\ Texas; CSU, California State University; SUNY, State \\ University of New York; CUNY, City University of \\ New York \\ * Enrollment Fall 2016 retrieved from \\ http://www.utrgv.edu/sair/.
}

\subsection{Messaging behaviors}

For each message, we determined whether it displayed one of four predefined behaviors: seeking help, offering support, humor, or bullying. We defined bullying behavior according to terms in a recent metaanalysis on cyberbullying. A message was considered to be bullying if it intended harm, was indicative of a power imbalance, and if the sender repeatedly sent these messages [16] We also consulted a team of three undergraduate students to identify topics that were relevant to undergraduates. Two undergraduate raters independently coded the selected messages for these four behaviors.

\subsection{Message topics}

We applied Latent Dirichlet allocation (LDA) to the message corpus to identify themes within the message content. LDA is a common method to categorize topics and themes [17]. Each topic, in turn, 
is probabilistically associated with various words. Since topics are defined purely in statistical terms, the user chooses its semantic interpretation (i.e., its label) on the basis of word probabilities for the topic.

Next, we sought to identify topics in which the LDA message classifications aligned most closely with human judgment. We did this with a subset of 1,200 randomly selected messages to which the LDA assigned a topic with a probability greater than 0.7 . For each of these messages, a team of three raters decided if the LDA topic assignment was correct (i.e., does the message discuss topic $\mathrm{X}$ ). Based on these results, we selected the four topics with the highest classification accuracies: relationships/sex, college living, politics, and school/classes.

In the final step, two undergraduate raters independently applied the four-topic classification scheme to 90 randomly selected messages. We found that their inter-rater agreement was high (Cohen's kappa $=0.78)$, so all remaining messages were coded by one of the two raters.

\subsection{Message popularity}

We determined the popularity of a message by the aggregate score of +1 votes (upvotes) and -1 votes (downvotes) assigned by Yik Yak users prior to data collection. Notably, if a message on Yik Yak reaches a sum score of -5 , it is automatically deleted from the social network. Thus, the lowest possible popularity score for a message in our data set was -4 .

\section{Results}

In all statistical analyses, the significance criterion was $\alpha=0.05$.

\subsection{Frequency of messaging behaviors}

$12 \%$ of messages were focused on one of the four categories: seeking help, offering support, humor, and bullying. Across states, we found significant differences in the relative frequency of messages offering support $(p<0.001)$ and bullying messages $(p$ $<0.001)$. We found no geographic differences for messages seeking help $(p=0.2)$ or for humorous messages $(p=0.4)$.

We found that the two states with the lowest rates of bullying, California and Florida, differed significantly from the states with the highest rates, New York and Texas (all $p<0.05)$.

\subsection{Frequency of topics}

To assess the relative frequency of topics discussed on Yik Yak, we used messages that raters uniquely assigned to one or to none of four LDAderived topics (relationships/sex, college living, politics, school/classes). This led to the exclusion of 117 messages $(0.7 \%)$ from the frequency analysis; $26.3 \%$ of the remaining messages dealt with either relationships and sex (14.9\%), college living (3.8\%), politics $(3.6 \%)$, or school and classes $(4.0 \%)$. In Table 2 , we break these numbers down further by state.

Using separate Fisher's exact tests, we found significant regional differences for each topic. New York had the fewest relationship messages and differed significantly from California and Texas $(p<0.001, p=$ 0.048).

We followed up on these significant effects with Bonferroni-corrected Fisher's exact tests for all pairwise comparisons between states for each topic. We found significant differences in the amount of college living messages between all states $(p<0.05)$, except for California and Texas, the two states with the most college living messages $(p=1.0)$. Finally, we found significant differences in the frequency of school-related messages between states $(p<0.05)$, with the exception of California and Texas, where school was discussed the most.

Table 2. Frequency of Messaging Behaviors and Topics by State

\begin{tabular}{r|r|r|rr}
\hline \multicolumn{6}{c}{ State } \\
\hline$N(\%)$ & $N(\%)$ & $N(\%)$ & $N(\%)$ & Fisher's \\
\hline
\end{tabular}

Messaging Behaviors

Seeking

help $\quad 70(1.6) \quad 94(2.0) \quad 65(1.5) \quad 70(2.0) \quad 0.2$

$\begin{array}{llllll}\text { Support } & 183(4.2) & 381(8.1) & 234(5.5) & 88(2.5) & <0.001\end{array}$

$\begin{array}{llllll}\text { Bullying } & 61(1.4) & 68(1.5) & 98(2.3) & 93(2.7) & <0.001\end{array}$

Humor $140(3.1) \quad 134(2.9) \quad 144(3.4) \quad 98(2.8) \quad 0.4$

Topics

$\begin{array}{llllll} & & & 562(13 . & 535(15 . & \\ \text { Relations } & 730(16.4) & 689(14.8) & 2) & 4) & <0.001 \\ \text { Living } & 224(5.0) & 83(1.8) & 157(3.7) & 180(5.2) & <0.001 \\ \text { Politics } & 133(3.0) & 122(2.6) & 317(7.5) & 35(1.0) & <0.001 \\ \text { Classes } & 208(4.7) & 114(2.4) & 150(2.5) & 198(5.7) & <0.001\end{array}$

CA, California; FL, Florida; NY, New York; TX, Texas

\subsection{Popularity of messaging behaviors}

In this and the following section we report findings on the popularity of the different messaging behaviors and topics, based on the aggregate of +1 votes (upvotes) and -1 votes (downvotes) each message 
elicited from Yik Yak users. We refer to this total as the popularity score of a message. In order to protect our analyses from the influence of a few massively popular messages, we flagged messages with a score greater than three standard deviations above the grand mean. We identified $305(1.8 \%)$ messages as popularity outliers and exclude these from further analysis.

Table 3 displays the mean popularity scores for the four messaging behaviors (seeking help, offering support, bullying, and humor) at the state level (CA, FL, NY, and TX). We submitted the individual message scores to a State $\mathrm{x}$ Behavior analysis of variance (ANOVA). Both main effects were significant: $F(3,1940)=5.11, M S E=4.1, p=0.002$ for State, and $F(3,1940)=25.19, M S E=4.1, p<$ 0.001 , for Behavior. The interaction between the two factors was not significant $(F(9,1940)=1.16, M S E=$ 4.1, $p=0.319$ ).

We used Tukey's range test to determine which state exhibited significantly different mean popularity scores. This analysis revealed that, on average, Yik Yak messages received lower popularity scores in Texas than in Florida and New York (both $p<0.05$ ). Additionally, Tukey's test showed that bullying messages were the least popular and differed significantly from messages seeking help, offering support, or humorous messages (all $p<0.01$ ). By contrast, humorous messages were the most popular and scored significantly higher than the other three message types (all $p<0.001$ ).

Table 3. Popularity of Messaging Behaviors and Topics by State

\begin{tabular}{|c|c|c|c|c|c|c|c|c|}
\hline \multicolumn{9}{|c|}{ State* } \\
\hline & \multicolumn{2}{|c|}{ CA } & \multicolumn{2}{|c|}{ FL } & \multicolumn{2}{|c|}{ NY } & \multicolumn{2}{|c|}{ TX } \\
\hline & $M$ & $S E$ & $M$ & $S E$ & $M$ & $S E$ & $M$ & $S E$ \\
\hline & \multicolumn{8}{|c|}{ Messaging Behaviors } \\
\hline Seeking & 1.04 & 0.26 & 1.37 & & & 0.30 & 0.53 & 0.27 \\
\hline Support & 1.00 & 0.11 & 0.98 & 0.08 & 1.22 & 0.12 & 0.77 & 0.16 \\
\hline Bul & 0.40 & 0.32 & 0.32 & 0.17 & 0.59 & 0.23 & 0.32 & 0.18 \\
\hline Humor & 1.50 & 0.20 & 1.71 & 0.22 & 2.14 & 0.27 & 1.27 & 0.20 \\
\hline & \multicolumn{8}{|c|}{ Topics } \\
\hline Relat & 1.56 & 0.09 & 1.03 & 0.08 & 1.16 & 0.10 & 0.96 & 0.08 \\
\hline Livi & 1.31 & 0.15 & 1.56 & 0.26 & 1.70 & 0.23 & 0.78 & 0.14 \\
\hline Politics & 1.17 & 0.21 & 1.46 & 0.24 & 1.34 & 0.14 & 1.49 & 0.43 \\
\hline Classes & 0.84 & 0.12 & 1.09 & 0.20 & 1.08 & 0.18 & 0.43 & 0.09 \\
\hline
\end{tabular}

Note. Mean message popularity scores are based on the aggregate number of upvotes $(+1)$ and downvotes $(-1)$ per-message.

CA, California; FL, Florida; NY, New York; TX, Texas

$*_{n}$ for each behavior and topic available upon request. We were unable to fit this information in the table.

\subsection{Popularity of topics}

Table 3 summarizes the mean popularity scores of messages that discussed one of the four topics identified through LDA: relationships and sex, college living, politics, or school and classes. A State (CA, FL, NY, TX) x Topic ANOVA revealed main effects of $F(3,4293)=11.23, M S E=4.9, p<0.001$ for State, and $F(3,4293)=6.03, M S E=4.9, p<0.001$ for Topic as well as a significant State-by-Topic interaction of $F(9,4293)=2.95, M S E=4.9, p=.002$. We carried out Tukey's test to further investigate the significant main effects. We found that Texas, the state with the lowest popularity scores overall, differed significantly from California, Florida, and New York (all $p<0.05$ ). Regarding the popularity of topics, school and classes was a significantly less popular topic than relationships and sex, college living, and politics (all $p<0.01$ ).

The significant State-by-Topic interaction indicates that states differ with respect to the relative popularity of topics. In order to identify patterns of topic popularity within each state, we conducted ANOVAs with Topic as a single factor, separately for each state. These ANOVAs yielded a significant effect of Topic for California, $F(3,1231)=5.36, M S E=5.39$, $p=0.001$, and Texas, $F(3,928)=5.84, M S E=3.17, p$ $<0.001$, but not for Florida, $F(3,985)=2.41, M S E=$ $4.91, p=0.066$, or for New York, $F(3,1149)=2.34$, $M S E=5.7, p=0.072$. We followed up on the significant effects for California and Texas with Tukey's test. In California, school and classes were a less popular topic than relationships and $\operatorname{sex}(p<$ 0.001 ). In Texas, messages about school and classes were less popular than messages about relationships and politics (both $p<0.01$ ).

\subsection{Interplay between variables}

In this section, we examine the relationship between the frequency of prosocial messages in which users sought help or offered support, the frequency of bullying messages, the popularity of these messaging behaviors, and the frequency of topics. We carried out analysis at the university level. For each university, we calculated mean messaging behavior frequencies, the corresponding

We found that schools with a greater frequency of help-seeking messages also exhibited a greater frequency of messages offering support. Campuses where students posted less about relationships and sex sent more messages offering support $(p<0.01)$. Moreover, messages offering support were more popular at higher-ranking schools $(\mathrm{p}<0.01)$. Second, bullying occurred more often on campuses where users 
posted more about politics $(\mathrm{p}<0.05)$. Finally, we found that the frequency of posts about classes was positively related to the frequency of posts about college living $(\mathrm{p}<0.05)$.

\section{Discussion}

Thanks to the growing popularity of social media across all segments of society, researchers have a plethora of data sources from which they can derive new insights about people's social and health-related attitudes, behaviors, and beliefs. The ability to observe social media users in near real-time holds particular promise in the domain of public health and health care, where rapid detection of health-relevant events and timely intervention are of the essence. The aim of the present study was to explore whether we could extract information pertaining to college students' health and well-being from their conversations on an Anonymous Social Network (ASN). To this end, we analyzed the frequency and popularity of prosocial messages and bullying messages as well as the frequency and popularity of topics discussed online.

In our data set, prosocial messages appeared more frequently than bullying messages (10.1\% vs. $1.9 \%)$, and there were significant regional differences in the frequency of messages associated with support or bullying. Notably, Yik Yak users attending Texas colleges sent the fewest supportive messages and the most bullying messages. We should interpret this finding with caution in light of the relatively small number of messages and universities considered for our study. Nevertheless, this finding highlights a potentially problematic pattern of social media use among college students that future research may link to adverse health outcomes. Unsurprisingly, bullying messages were the least popular and humorous messages were the most popular among Yik Yak users, independent of what state they lived in.

In order to identify the topics of Yik Yak messages, we relied on statistical modeling as an alternative to the subjective classification scheme recently used by Black and colleagues. ${ }^{15}$ A subsequent analysis of topic prevalence revealed that relationships/sex was the most frequently discussed topic among college students. School and classes turned out to be the least popular topic, as measured by the number of up- and downvotes a message received. From an intervention point of view, regional differences in topic frequency and popularity matter because they offer campus representatives and health professionals clues on how to best engage a student population both online and offline. Although the relative popularity of topics was similar across states, we found greater regional variation in the relative frequency of topics. For example, 7.5\% of Yik Yak messages in the state of New York discussed politics compared to only $1 \%$ in Texas, and college living was addressed in $5 \%$ of messages in California, but in only $1.8 \%$ of messages in Florida.

With our final correlational analysis we wanted to learn more about factors that promote prosocial online behaviors and prevent cyberbullying at U.S. colleges. Several findings are worth noting. At schools where students often sought help through messages, messages offering support were also more frequent. We speculate that students may offer support in response to requests for help, but the reverse relationship is also conceivable: At schools where support is offered frequently, students may feel encouraged to ask for help. A higher prevalence of supportive messages also appears to be a characteristic of higher-ranking universities. It is more difficult to interpret why messages of support were sent more often at schools where relationships and sex were discussed less frequently. This requires further investigation. It is also not clear why there was a positive relationship between the popularity of messages offering support and the frequency of the school/classes topic.

Two results speak directly to the frequency of cyberbullying on college campuses. First, there was a positive relationship between bullying and the popularity of messages seeking help. One interpretation for this finding is that students react prosocially to a higher prevalence of bullying by encouraging help-seeking behavior, although they did not appear to actually offer more support (the correlation between the frequency of supporting and bullying messages was negative and not significant). An alternative hypothesis is that certain prosocial messaging behaviors can trigger cyberbullying. Additionally, students at schools with higher incidence of bullying frequently discussed politics.

\section{Conclusion}

This study has strong implications for the education public health and broader field of health care. Educators could use similar methods to find topics that may be engaging to students on campus. In particular, campus administrators and health services units could identify topic areas where students could engage in a campus-wide dialogue. This could also be helpful for public health professionals because it would provide insight into campus conversations that were leading bullying or hostility. Educators and clinicians could work together to foster a healthier dialogue around the subject and encourage a campus culture of reaching out to fellow students to offer support. In 
addition to gaining insights on conversations on college campuses, this study represents a first step in guiding research focused on ASNs. The results of this study can help promote the labelling and mining of social data to help students, parents, administrators, and healthcare workers identify cyberbullying and design interventions to stop it.

This type of work naturally presents opportunities to computer scientists working in health services as well. Mining data from ASNs can extend beyond the college campus and to the public. Computer scientists can design tools to mine and categorize public social data and help create an even farther reaching monitoring system for educators and public health professionals.

The major limitations of this study include the small number of colleges and universities considered, the lack of ability to generalize as Yik Yak has closed down since this study was conducted, and the modest number of Yik Yak messages per school. We therefore caution against generalizing our findings until they can be replicated with larger samples and on other ASN's. The main intention of this study was to understand students' online behaviors and interests from their posts on an ASN and, more specifically, to garner initial insight into conditions affecting prosocial and antisocial uses of social media that could be integrated in health services. We believe that the findings reported here can be a stepping stone to further research on this topic as well as differences in health behaviors and risks communicated on ASN's versus non-ASN's.

\section{References}

[1] K. Vail-Smith, W.M. Felts, and C. Becker. "Relationship between sleep quality and health risk behaviors in undergraduate college students.", College Student Journal, 2009, 43, pp. 924.

[2] P.G Williams, and T.L. Moroz. "Personality vulnerability to stress-related sleep disruption: Pathways to adverse mental and physical health outcomes", Personality and Individual Differences, 2009, 46, pp. 598-603.

[3] N. Galambos, A. Dalton, and J. Maggs. "Losing sleep over it: daily variation in sleep quantity and quality in Canadian students' first semester of university", Journal of Research on Adolescence, 2009, 19, pp. s741-761.

[4] B.M. Roane, R. Seifer, K.M. Sharkey, E. Reen, T.L. Bond, T. Raffray, and M.A. Carskadon. What role does sleep play in weight gain in the first semester of university?" Behavioral Sleep Medicine, 2014, 13, pp. 491-505.
[5] G. Curcio, M. Ferrara, and L. Gennaro. "Sleep loss, learning capacity and academic performance", Sleep Medicine Reviews, 2006, 10, pp. 323-337.

[6] S. Bauman, R.B. Toomey, and J.L. Walker. "Associations among bullying, cyberbullying, and suicide in high school students", Journal of Adolescence, 2013, 36, pp. 341-350.

[7] M. Gámez-Guadix, I. Orue, P.K. Smith, and E. Calvete. "Longitudinal and reciprocal relations of cyberbullying with depression, substance use, and problematic internet use among adolescents, Journal of Adolescent Health, 2013, 53, pp. $446-452$.

[8] J. Wang, T.R. Nansel, and R.J. Iannotti. "Cyber and traditional bullying: Differential association with depression", Journal of Adolescent Health, 2011, 48, pp. 415-417.

[9] S.D Young, C. Rivers, B. Lewis "Methods of using realtime social media technologies for detection and remote monitoring of HIV outcomes", Preventive Medicine, 2014, $63,112-5$.

[10] M. De Choudhury, M. Gamon, S. Counts, and E. Horvitz. "Predicting depression via social media", ICWSM, 2013.

[11] J. Mahler. "Who spewed that abuse? Anonymous Yik Yak app isn't telling”, The New York Times, 2015, www.nytimes.com/2015/03/09/technology/popular-yik-yakapp-confers-anonymity-and-delivers-abuse.html. Accessed November 21, 2016.

[12] A. Shontell. "Why a girl who was viciously bullied on Yik Yak now believes in the anonymous app's future", 2015, www.businessinsider.com/elizabeth-long-was-bullied-onyik-yak-2015-3. Accessed November 22, 2016.

[13] N. Valencia. "Yik Yak chat app stirring up trouble in high schools", 2014, www.cnn.com/2014/03/07/tech/yikyak-app-high-school-problems/. Accessed November 14, 2016.

[14] G. Wang, B. Wang, T. Wang, A. Nika, H. Zheng, and B. Zhao. Whispers in the dark: analysis of an anonymous social network. Proceedings of the 14th Internet Measurement Conference (IMC), 2009, pp.137-150.

[15] E. Black, E, K. Mezzina, and L. Thompson. "Anonymous social media-understanding the content and context of Yik Yak", Computers in Human Behavior, 2016, 57, pp. 17-22.

[16] R. Garett, L.R. Lord, and S.D. Young. "Associations between social media and cyberbullying: a review of the literature", Mhealth [Internet], 2016, 2. Available from: https://www.ncbi.nlm.nih.gov/pmc/articles/PMC5344141/ 
[17] Blei, D.M., Ng, A.Y., Jordan, M.I. (2003). Latent dirichlet allocation. Journal of Machine Learning Research, 3, 993-1022.
[18] Wall Street Journal/Times Higher Education College Rankings 2017. (2016). www.timeshighereducation.com/rankings/united-states/2017. Accessed 9

December 